Signal \& Image Processing: An International Journal (SIPIJ) Vol.10, No.4, August 2019

\title{
RANSAC BASED MOTION COMPENSATED RESTORATION FOR COLONOSCOPY IMAGES
}

\author{
Nidhal Azawi and John Gauch \\ Department of Computer Science and Computer Engineering, \\ University of Arkansas, Fayetteville, Arkansas
}

\begin{abstract}
Colonoscopy is a procedure that has been used widely to detect the abnormality in a colon. Colonoscopy images suffer from a lot of problems that make it hard for the doctor to investigate/ understand a colon patient. Unfortunately, with the current technology, three is no way for doctors to know if the whole colon surface has been investigated or not. We have developed a method that utilizes RANSAC-based image registration to align sequences of any length in the colonoscopy video and restores each frame of the video using information from these aligned images. We proposed two methods. First method used the deep neural net for the classification of informative and non-informative image. The classification result was used as a preprocessing for alignment method. Also, we proposed a visualization structure for the classification results. The second method used the alignment to decide/classify the bad and good alignment by using two factors. The first factor is the accumulated error and the second factor contain three checking steps that check the pair error alignment beside the geometry transform status. The second method was able to align long sequences.
\end{abstract}

\section{KEYWORDS}

Visualization, RANSAC, sequence length, geometry transform, classification, Colonoscopy.

\section{INTRODUCTION}

The main goal of this paper is to remove specular highlights and shiny areas in colonoscopy images. Colonoscopy images suffer from many problems. Some of these problems/issues are blurring which caused by either the camera is very close to the colon surface or some dirt on the lens that prevent the doctor to see things clearly. Another problem is the existence of the specular highlights which are vary from one image to another based on the amount of light reflected from the colon surface to the camera which has been illustrated in detail in our previous two papers.

We used image registration as a tool to help to remove some unwanted artefacts from colonoscopy images. We addressed the problem of colonoscopy image registration in [1], [2]. The proposed approach relies on three pre-processing steps. These three processing steps are the removal of non-informative images, median and mean filtering with or without image resizing and image resizing. To the best of our knowledge we are the first researcher who tested these three pre-processing steps in image registration for colonoscopy images. We were able to restore and enhance image details in colonoscopy by creating an image panorama from registered images. The experimental results show that the removal of non-informative images allows enhancing the alignment results. 
Signal \& Image Processing: An International Journal (SIPIJ) Vol.10, No.4, August 2019

In this paper, we have modified our approach by excluding the preprocessing classification phase and instead we used another approach to classify bad and good aligned images. We used two factors to control the process of aligning sequence of images. First factor by computing the accumulated error after and before alignment to control the process of aligning sequence of images beside relying on the three condition in our previous approach to automatically exclude the unwanted images that cannot be aligned with together. This approach can speed up the algorithm implementation by omitting the classification phase and let the alignment algorithm decide the good and bad aligned images.

A lot of work has been done to align two or more than one image and we are going to mention some of them. Image registration/alignment has been used in many fields such as computer vision, medical image processing, motion tracking and many others. For this reason, a lot of work has been done to develop fast and efficient image alignment methods [2].

Image registration has been defined in many ways. Some authors defined the image registration is the process of matching common features in order to align two or more images. Other authors mentioned that image alignment is the way of identifying an optimal transformation that can fit or give the best transformation for a particular input image [3][4]. These images can be taken from different viewpoints. Image registration-based feature matching involves feature detection and extraction, feature matching, transformation and fitting function, and image resampling and transformation. Image registration can integrate information from a sequence of image.

Image alignment methods can be classified into two broad categories based on how images are aligned with each other. The first category is area-based matching. A large number of methods have been devised for comparing patches, and for looking for (displacements with different accuracy/speed trade-offs. Recent examples of area-based approaches are described by [2] [5], [6], [7] and [8].

A proposed work in 2018 has been implemented by [9]. Authors used two phases to resolve the specular highlight existence in the colonoscopy images. First phase is to detect the specular highlight and the second phase purpose is to remove the specular by using inpainting techniques. Alignment based feature matching is the second category. In this category, image is examined to find keys points and describe the area surrounding each key point to create a feature vector that can be matched against feature vectors from another image. Some of Key points examples are corners, centres of bright/dark objects and blobs. Feature descriptors are chosen so they describe the local neighborhood of feature points in a way that is robust to changes in position, orientation, scale, and illumination. The scale invariant feature transform (SIFT) is very popular method that used feature-based image alignment techniques [10]. Other recent examples of this approach include [11], [12] and [13].

Two stages scheme has been proposed by [14]. It consists of First, directional mapping to normalize images and to mitigate the effect of saturation has been implemented. Second, intensity invariant features have been represented using LBP (a non-parametric local binary pattern). The experimental results showed that their method achieved better accuracy than the state-of-the-art methods. A robust approach against non-uniform illumination method has been done in image alignment based on matching of relative gradient map [15]. Images that are seen or captured from different viewpoints has been used in [16]. The author mentioned that their approach has the ability to distinguish between square and rectangle while affine invariant approaches could not recognize them. 
Signal \& Image Processing: An International Journal (SIPIJ) Vol.10, No.4, August 2019

Image registration has been used a lot in medical image analysis including many applications. Application areas include computer aided diagnosis, surgery simulation, intervention and treatment planning, radiation therapy, anatomy segmentation, computational model building, and contrast enhanced based approach, partial volume corrections based on CT images, and assisted/guided surgery. Medical image registration has been applied on a wide range of body images, see [17], [18], [19], [20] and [2].

The rest of the paper is organized as follows. Section 2 presents our two proposed methods in detail with the experimental results. The conclusion and future work are in Section3.

\section{OUR APPROACH}

\subsection{Method1}

The method we used to find and remove bad images is based on feature-based image classification described in our earlier paper [1]. Two approaches have been proposed with RANSAC image alignment to align colonoscopy images. We created two visualization structure for each approach.

For clarification, we used RANSAC (random sample consensus) To register sequences of $\mathrm{N}$ colonoscopy images to each other to solve for the projective transformation that produces the best image alignment. We used pairwise and $\mathrm{N}$ sequence alignment methods. RANSAC is a fitting model to some data in the presence of outliers and it uses trial and error approach to find model parameters that best fit the data. For more information see [2], [21].

Projective homography matrix has eight DOF (degree of freedom) and the affine homography matrix has six DOF. Comparison between the projective and affine transformation and more information about them can be found in [22], [23], [2].

Using RANSAC to align colonoscopy images with affine transformations yields a large number of nonsingular transformation matrices, which means there is no viable affine transformation that can successfully align these two images. Hence affine transformations are not a good choice for image registration. Since the camera capturing colonoscopy video is changing position during the procedure, we will use RANSAC to calculate the best projective transformation that aligns all pairs of images within a moving 10 frame window of the colonoscopy image. The algorithm we use to register, and process colonoscopy images has the following steps [2]:

Step1: Classify images - Images have been automatically classified as being informative or noninformative using feature-based image classification [1].

\section{Step2:}

- $\quad$ Loop over all sets of 10 consecutive images in the colonoscopy video.

- $\quad$ Detect and extract features points for all 10 images in the sequence.

- $\quad$ Find the matching feature points for all pairs of images.

- $\quad$ Find the best projective transformation using RANSAC algorithm as follow: -

a. Including all transforms that satisfy all conditions below:

1- The number of inliers points greater than 5 points.

2- $\quad$ The determinant of the transform greater than 0.5 .

3- The image difference after alignment is less than before alignment.

- $\quad$ Save aligned images.

- Create Panorama. 
Signal \& Image Processing: An International Journal (SIPIJ) Vol.10, No.4, August 2019

\subsubsection{Method1 Experimental Results}

A preprocessing step to image panorama creation is used in [2]. The output resulted from the preprocessing method is used as input to our alignment method. The best alignment with the 10 subsequent images has been calculated for each frame. We set the maximum sequence length to 11. Figure 1 shows that in some cases only a subset of the 10 subsequent images were able to be successfully aligned with the starting image.

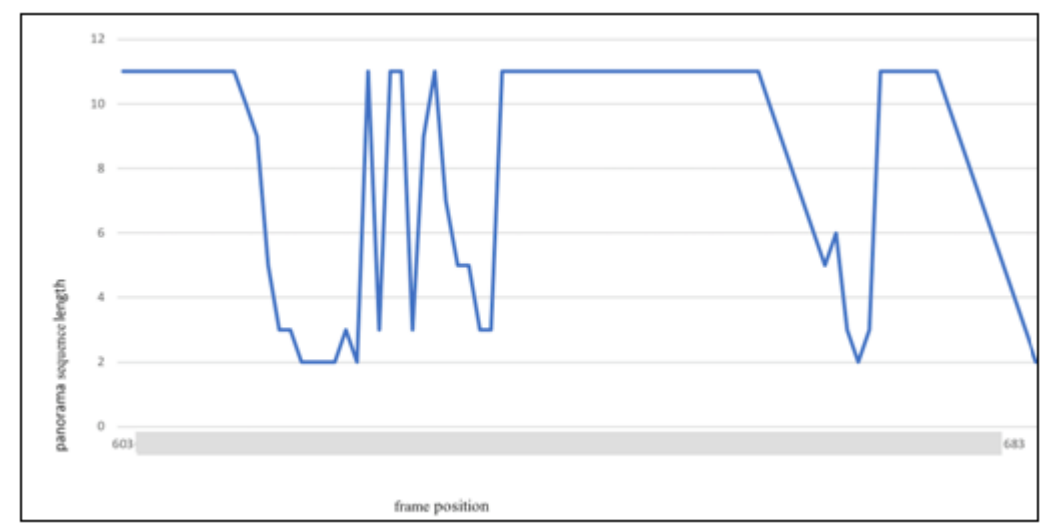

Figure 1. illustration of panorama sequence length for colonoscopy frames [2].

We had a RMSE of 4.16 and a percentage aligned of $37 \%$ for the original video but after excluding the non-informative images using classification phase, the RMSE increased slightly to 4.38 and the percentage aligned increased significantly to $48 \%$.

The average CPU time for image alignment and panorama creation was also significantly reduced when non-informative images were excluded. This is because the RANSAC method execute the maximum number of iteration when trying to align no informative images. The results from our image sequence alignment and panorama creation are illustrated in the figures below. In figure 2, we show how bad subsequences produce very poor panoramas that has no useful information. Enhanced version examples can be found in figure 3.
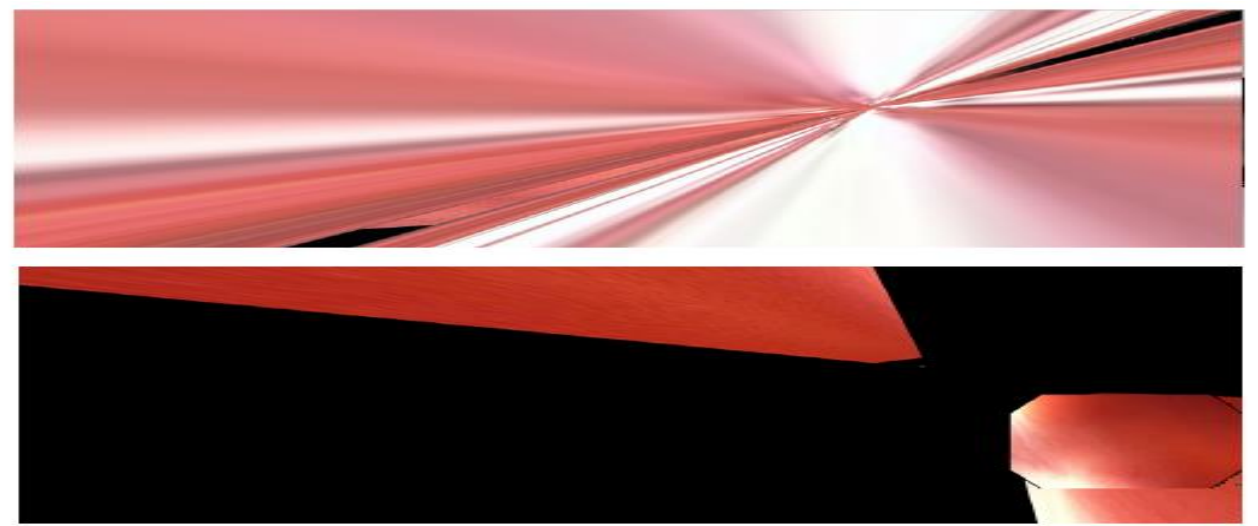

Figure 2. Examples of unsuccessful panorama creation. panoramas are bad because they are been generated from highly distorted images [2]. 
Signal \& Image Processing: An International Journal (SIPIJ) Vol.10, No.4, August 2019
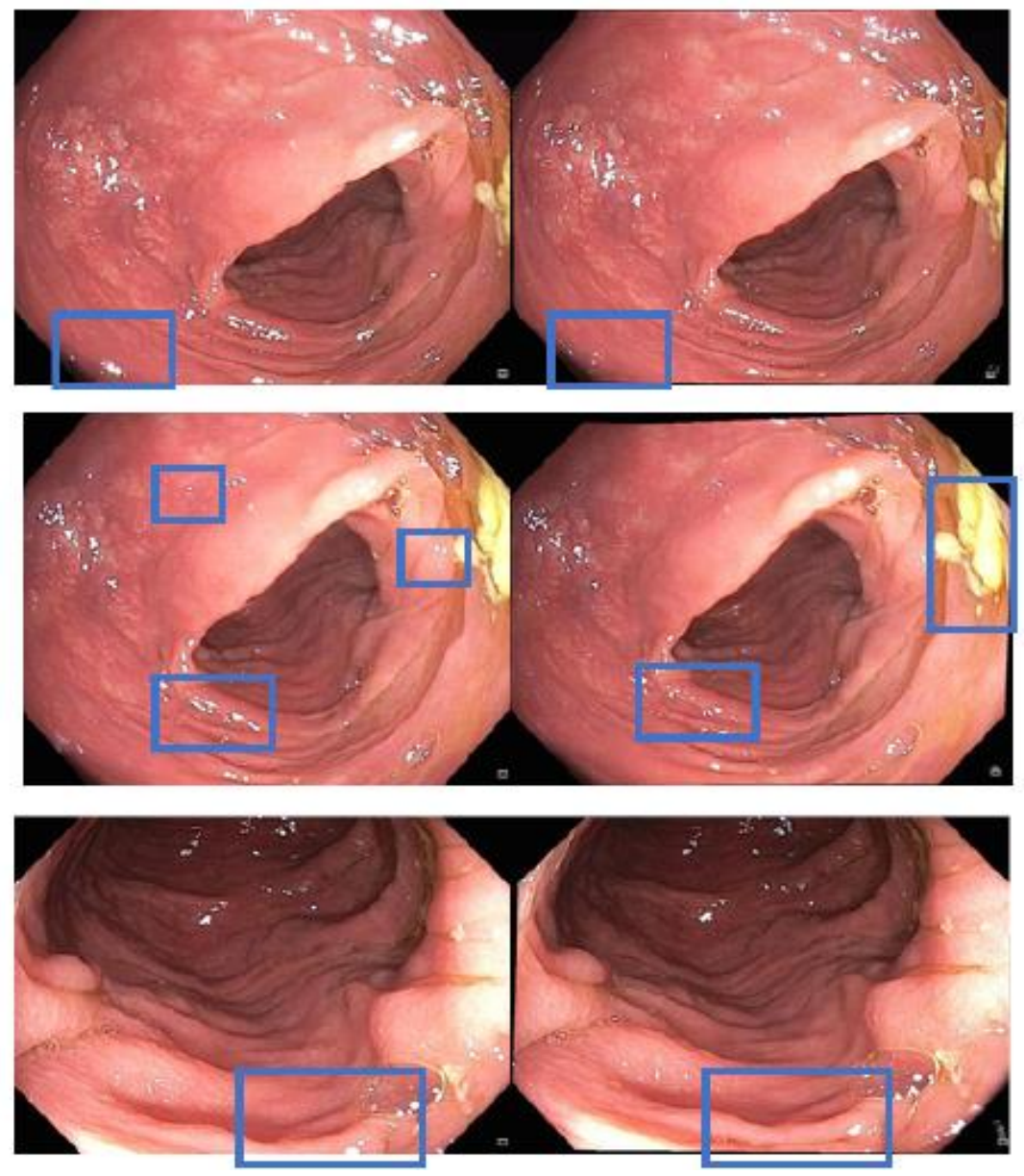

Figure 3. Examples of panoramas for image sequences that had zooming out motions. The images on the right are the corresponding panoramas and the images on the left are the original colonoscopy images. Blue boxes indicate areas of where specular highlights have been removed and where more image detail is visible [2].

This method helps to reduce the specular highlight, but the computation time grows with the existence of bad/noninformative images.

\subsection{Method2}

Excluding the classification phase can reduce the computational time required to implement our RANSAC algorithm. Method2 steps can be listed as follows: -

- Calculate the accumulated error after and before alignment.

- Do the following if the accumulated error after alignment is less than before alignment

o Use step2 in method1

- Save the aligned sequences and create sub videos. 
Signal \& Image Processing: An International Journal (SIPIJ) Vol.10, No.4, August 2019

The accumulated error is resulting from adding the error generated in each alignment step. The resulted alignment sequence automatically specified and controlled by calculation the total alignment error. Hence, we did not need to specify the maximum length in this experiment. In this way, the bad and good aligned images have been classified automatically. After we implemented Method2, the results showed that the aligned sequence length varies for each frame and sometimes it exceeded 300 images.

\section{CONCLUSION AND FUTURE WORK}

We illustrated our methods for motion compensated colonoscopy image restoration using RANSAC-based image registration. Method1 uses preprocessing steps to align sequences of 11 consecutive images(method1) while method2 exclude the preprocessing steps to align sequences of various consecutive images length in the colonoscopy video.

In method1, our experiments verify that the removal of non-informative images prior to image registration reduces the CPU time necessary for image alignment and it helps to generate some good panoramas for good images.

In method2, we tried to use the alignment algorithm to specify the good sequence length instead of using machine learning algorithm. Method 2 helps to reduce the computation time and it also able to align a long sequence of consecutive images.

For future work, we will focus on enhancing and improving the quality of image alignment by reducing the CPU time using different parallel programming and image registration techniques.

\section{REFERENCES}

[1] N. Azawi, J. Gauch, "Automatic Method for Classification of Informative and Noninformative Images in Colonoscopy Video", Int. Conf. on Medical Image Processing and Analysis (ICMIPA), Vancouver, Canada, August 2018.

[2] N. Azawi and J. Gauch, "MOTION C OMPENSATED RESTORATION OF COLONOSCOPY VIDEO," pp. 243-256, 2019.

[3] L. Dung, C. Huang, and Y. Wu, "Implementation of RANSAC Algorithm for Feature-Based Image Registration," Journal of Computer and Communications, pp. 46-50, 2013.

[4] F.P.M. Oliveira, J.M.R.S. Tavares. Medical Image Registration: a Review. Computer Methods in Biomechanics and Biomedical Engineering 17(2):73-93, 2014.

[5] S. B. Kang, M. Uyttendaele, S. Winder, and R. Szeliski, "High dynamic range video," ACM Trans. Graph., vol. 23, no. 3, pp. 319-325, 2003.

[6] F. M. Candocia, "On the Featureless Registration of Differently Exposed Images," in Proc. Int. Conf. Imaging Science, Systems \& Technology, Las Vegas, NV, USA, Jun. 2003, vol. I, pp. 163-169.

[7] Hossain and B. K. Gunturk, "High Dynamic Range Imaging of Non-Static Scenes," in Proc.SPIE Digital Photography VII, 2011, vol. vol. 7876. 
Signal \& Image Processing: An International Journal (SIPIJ) Vol.10, No.4, August 2019

[8] H. Q. Luong, B. Goossens, A. Pizurica, and W. Philips, "Joint photometric and geometric image registration in the total least square sense," Pattern. Recognition. Lett., vol. 32, no. 15, pp. 20612067, 2011.

[9] O. El Meslouhi, M. Kardouchi, H. Allali, T. Gadi, and Y. A. Benkaddour, "Automatic detection and inpainting of specular reflections for colposcopic images," Open Comput. Sci., vol. 1, no. 3, pp. 341$354,2011$.

[10] D. G. Lowe, "Object recognition from local scale-invariant features," Proc. of the Int. Conf. on Computer Vision, pp. 1150-1157, 1999.

[11] B. Zitova and J. Flusser, "Image registration methods: A survey," Image Vis. Computer, vol. 21, pp. 977-1000, 2003.

[12] S. Oldridge, G. Miller, and S. Fels, "Mapping the problem space of image registration," in Proc. Can. Conf. Computer and Robot Vision, St. John's, NF, Canada, May 2011, pp. 309-315.

[13] M. Tico and K. Pulli, "Robust image registration for multi-frame mobile applications," in Proc. Asilomar Conf. Signals, Systems \& Computers, Pacific Grove, CA, USA, 2010, pp. 860-864.

[14] S. Wu, Z. Li, J. Zheng, and Z. Zhu, "Exposure-robust alignment of differently exposed images," IEEE Signal Process. Lett., vol. 21, no. 7, pp. 885-889, 2014.

[15] S. Wu, Z. Li, J. Zheng, and Z. Zhu, "Exposure-robust alignment of differently exposed images," IEEE Signal Process. Lett., vol. 21, no. 7, pp. 885-889, 2014.

[16] S. Wei and S. Lai, "Robust and efficient image alignment based on relative gradient matching," IEEE Trans. image Process., vol. 15, no. 10, pp. 2936-43, 2006.

[17] C. Wu, B. Clipp, X. Li, J. M. Frahm, and M. Pollefeys, "3D model matching with viewpointinvariant patches (VIP)," 26th IEEE Conf. Comput. Vis. Pattern Recognition, CVPR, pp. 1-8, 2008.

[18] P. A. Freeborough and N. C. Fox, "Modelling Brain Deformations in Alzheimer Disease by Fluid Registration of Serial 3D MR Images", vol. 22. 1998.

[19] D. Leow, A. D. Klunder, C. R. Jack, A. W. Toga, A. M. Dale, M. A. Bernstein, P. J. Britson, J. L. Gunter, C. P. Ward, J. L. Whitwell, B. J. Borowski, A. S. Fleisher, N. C. Fox, D. Harvey, J. Kornak, N. Schuff, C. Studholme, G. E. Alexander, M. W. Weiner, and P. M. Thompson, "Longitudinal stability of MRI for mapping brain change using tensor-based morphometry," Neuroimage, vol. 31, no. 2, pp. 627-640, 2006.

[20] K. A. Ganser, H. Dickhaus, R. Metzner, and C. R. Wirtz, "A deformable digital brain atlas system according to Talaicrach and Tournoux,” Med. Image Anal., vol. 8, no. 1, pp. 3-22, 2004.

[21] X. Huang, J. Ren, G. Guiraudon, D. Boughner and T. M. Peters, "Rapid Dynamic Image Registration of the Beating Heart for Diagnosis and Surgical Navigation," in IEEE Transactions on Medical Imaging, vol. 28, no. 11, pp. 1802-1814, Nov. 2009. doi:10.1109/TMI.2009.2024684.

[22] R. Redzuwan, N. A. M. Radzi, N. M. Din, and I. S. Mustafa, "Affine versus projective transformation for SIFT and RANSAC image matching methods," 2015 IEEE Int. Conf. Signal Image Process. Appl., pp. 447-451, 2015.

[23] O. El Meslouhi, M. Kardouchi, H. Allali, T. Gadi, and Y. A. Benkaddour, "Automatic detection and inpainting of specular reflections for colposcopic images," Open Comput. Sci., vol. 1, no. 3, pp. 341354, 2011. 
Signal \& Image Processing: An International Journal (SIPIJ) Vol.10, No.4, August 2019

\section{AUTHORS}

Nidhal K. Azawi finished her bachelor and master's degree in computer science / image processing at the University of Technology / department of computer science in Baghdad. She was an assistant teacher in the computer science and Engineering in the University of Al_Mustansiriya in Baghdad. She is now a PhD student researcher in computer science and engineering department in the University of Arkansas. Her research is in image processing and computer vision application, biomedical image enhancement, and motion tracking applications. Her research interest seeks to combine machine learning with image processing and computer vision for advancements in the medical field pertaining to image enhancement, reconstruction, motion analysis, and visualization.

John M. Gauch joined the computer science and computer engineering department at the University of Arkansas as a professor in 2008. He held previous faculty positions at the University of Kansas and Northeastern University. His research interests include real-time digital video processing, content-based image and video retrieval, biomedical image enhancement, image segmentation, and motion tracking applications. This research has resulted in over sixty publications in these areas including one book and one patent. 